\title{
Brain responses of explicit and implicit memory: an event-related potential study
}

\author{
Ming-Jang Chiu ${ }^{\mathrm{a}}$, Mau-Sun Hua ${ }^{\mathrm{c}}$, Ta-Fu Chen ${ }^{\mathrm{a}}$, Hai-Gwo Hwu' ${ }^{\mathrm{b}}$, Chiun-How Kao and \\ Chun-Houh Chen ${ }^{\mathrm{d}}$
}

\begin{abstract}
Departments of ${ }^{\mathrm{a}}$ Neurology, ${ }^{\mathrm{b}}$ Psychiatry, National Taiwan University Hospital, College of Medicine, ${ }^{\mathrm{C}}$ Department of Psychology, National Taiwan University and ${ }^{\mathrm{d}}$ Institute of Statistics, Academia Sinica, Taipei, Taiwan
\end{abstract}

Correspondence and requests for reprints to Dr Ming-Jang Chiu, MD, PhD, No. 7, Sec. I, Zhong-Shan S. Rd, Taipei I00, Taiwan

Tel: + $886968661507 /+886223123456$ ext 5339; fax: +886223418395; e-mail: mjchiu@ntumc.org, mjchiu@ha.mc.ntu.edu.tw

Sponsorship: The study was sponsored in part by a grant from National Health Research Institute, Taiwan NHRI-EX94-9|13PP and the ERP device by Mental Health Foundation, Taiwan.

Received 28 June 2006; accepted 30 June 2006

\begin{abstract}
Implicit memory is acquired by an unintentional or unconscious learning. Recognition memory involves either automatic knowing or consciously controlled remembering. We provided an eventrelated potential paradigm capable of differentiating memory for the explicitly learned, implicitly learned and unstudied materials. In the explicit memory, we obtained both frontal (controlled retrieval) and parietal (recollection) old/new effects. In the implicit memory, we found persistent occipitotemporal activation (visual
\end{abstract}

priming) and late attenuation in the temporoparietooccipital (repetition suppression). Event-related potential provides an insight into the dissociable mechanism of memory function that supports the dual process model with an enhanced temporal resolution on the dynamic process of both explicit perceptual learning and implicit perceptual priming. NeuroReport 17:1483-1486 (c) 2006 Lippincott Williams \& Wilkins.

Keywords: event-related potentials, explicit memory, familiarity, implicit memory, knowing, perceptual priming, recognition memory, recollection, remembering

\section{Introduction}

Implicit memory is acquired by unintentional or unconscious learning [1]. Participants of implicit learning experiments could make decisions with better-than-chance accuracy but had little ability to describe the rule, context or source of the learned information. Recognition memory is a fundamental facet of our ability to remember. Current research supports theories claiming that recognition memory involves two components interpreted within a dualprocess independence model [2,3]. Knowing reflects an automatic form of memory and remembering reflects a consciously controlled form of memory [4].

The recognition memory has been studied by eventrelated potential (ERP) and a common finding of the ERP studies was the parietal old/new effect $[5,6]$. The majority of these studies have interpreted results from the perspective of dual-process models [7-9], although debates remained $[5,10]$. Some authors found it covaried with the number of accurate source judgments [11] while others did not agree $[4,7]$. In addition, some would attribute it to memory strength and decisional factors [12]. On the other hand, the frontal old/new effect was less well recognized or less comprehensively investigated than its parietal counterpart. The frontal old/new effect was considered to be related to familiarity and the effect was similar for words and non-words [13]; and it did not differ between deeply and shallowly encoded words [8].
In this study, we provided an ERP paradigm capable of differentiating memory for the explicitly learned, implicitly learned and unstudied materials. We provided either explicit perceptual learning or implicit perceptual priming without conceptual encoding [9]. We aimed to test the dualprocess hypothesis of recognition memory and to explore the neural correlates of these posited dissociable mechanisms with a temporal resolution superior to functional imaging currently available.

\section{Methods}

\section{Participants}

Twenty-five right-handed study participants (mean age of $29.9 \pm 4.6$ years and mean education years 16.6 \pm 2.0 ), colleagues or students working or studying at the university hospital, took part in the behavioral tasks. Fifteen of the 25 participants were female. Another 25 participants (mean age of $26.7 \pm 6.9$ years, mean education years $16.5 \pm 1.9$ and 15 of them were female) with similar backgrounds were recruited for the ERP study. The study was approved by the Institute Review Board of the university hospital. All participants gave informed consent.

\section{Stimuli}

Stimuli were figures consisting of a combination of simple geometry forms. We designed the pictorial stimuli in such a 
way as to make it difficult to generate verbal cues within the short display time given. After the 32 figures were constructed, they were randomly categorized into three sets of figures (eight, eight and 16, respectively) to equalize the stimuli in terms of complexity. The first eight figures were used for memorizing to test for the explicit memory. The other eight figures were used as the new (contrast) material during the first phase test for the explicit memory function and were also used as the targets of the implicit memory during the second phase test. The final 16 figures were used as the new material during the second phase test. All the stimuli were presented in a pseudorandom sequence such that the order of display is effectively a random sequence with the constraint of three identical consecutive figures at maximum.

\section{Procedure}

\section{Behavioral task}

During the learning phase, participants were presented with the first eight figures. Each figure was displayed for $2000 \mathrm{~ms}$ with repeated presentation six times each. Participants were instructed to memorize as many of these figures as possible. After an interval of $5 \mathrm{~min}$, we performed the first phase test in which participants were instructed to judge whether they remembered the figures presented and to press a button representing either a yes or a no within an interval of $1500 \mathrm{~ms}$. This phase included a total of 96 presentations from 16 different figures, each being shown six times. After another 5-min break, we performed the second phase test in which participants were instructed to judge whether there was any familiarity with the figures presented and to once again press the button for their decision within an interval of $1500 \mathrm{~ms}$. In this phase, there were a total of 48 presentations (first set, eight figures two times + second set, eight figures two times + third set, 16 figures one time). All responses were recorded on a computer for further analysis. The correct rates were obtained by adding up the correctly accepted and the correctly rejected items. The stimuli were presented to the participants through a 15 -inch LCD screen at a distance of about $60 \mathrm{~cm}$; the figures were displayed in the size range of $4.5-5.5 \mathrm{~cm}$ in diameter, and hence a visual angle of $4.3-5.2^{\circ}$.

\section{Event-related potential task}

The presentation parameters and the instruction for the participants in the learning phase and in the first phase test were identical to those in the behavioral tasks. After a 5-min break, we performed the second phase test in which the figures were presented for $500 \mathrm{~ms}$ and then a blank screen was presented for $200 \mathrm{~ms}$. In this phase, there were a total of 144 displays (first set, eight figures six times + second set, eight figures six times + third set, 16 figures three times). During the second phase test, the participants were asked to keep their full attention on the very short display and to judge whether there was any familiarity with the figures presented. They were informed that the total duration of this phase was less than $2 \mathrm{~min}$.

ERPs were recorded with a 32-channel montage in a NeuroScan System (NuAmps, Compumedics, El Paso, Texas, USA), with a sampling rate of $1 \mathrm{kHz}$ and a bandpass of $0.1-100 \mathrm{~Hz}$. Evoked potentials were obtained from averages of 48 study blocks of each category. Two additional eye channels were used to detect ocular movements. Blocks contaminated by blinks or eye movement artifacts $(> \pm 75 \mu \mathrm{V})$ were removed from further analysis. In addition, visual inspection of all segmented epochs was performed to assist the pre-analysis artifact rejections. The artifact-free electroencephalogram segments were subjected to baseline adjustments with the mean amplitude of the whole segment $(-50$ to $700 \mathrm{~ms})$ and were then averaged over the same segment. Averaged reference was applied to obtain the scalp topography using double spline interpolation to minimize the reference-site effect [14]. ERP components were identified through the global field power curves (Fig. 1) [15]. Statistical analysis comparing areas under curve of the three different tasks were performed in every channel. Further computation of the between-task t-Maps was also performed. Positions and $t$ values were reported with the nomenclature of the 10-10 International System.

\section{Results}

No statistical significance was observed between the two groups of participants in sex, age $(t=-1.6, P=0.29$, d.f. $=48)$ and education years $(t=0.00, P=0.59$, d.f. $=48)$.

\section{Behavioral performance}

The mean correct rates were $96.7 \pm 4.3 \%$ for explicit memory and $96.8 \pm 2.9 \%$ for implicit memory in the behavioral tasks, and $96.2 \pm 9.7 \%$ for explicit memory in the ERP tasks. Objectively, there was no significant intergroup difference (explicit memory: $t=0.58, P=0.51$, d.f. $=48$ ) and no significant intertask difference (explicit versus implicit: $t=-2.54$, $P=0.80$, d.f.=24). Subjectively, the participants reported that they could mentally visualize the explicitly learned pictures but not the implicitly learned ones.

\section{Event-related potential performance}

The field potential changes through time represented by global field power bore similar basic structures in the three different tasks (Fig. 1). The first positive peak was around $150 \mathrm{~ms}$ followed by a prominent negativity at $400 \mathrm{~ms}$ and then a positive slow component. We computed the areas under the curve for the four segments, namely, 100-200,

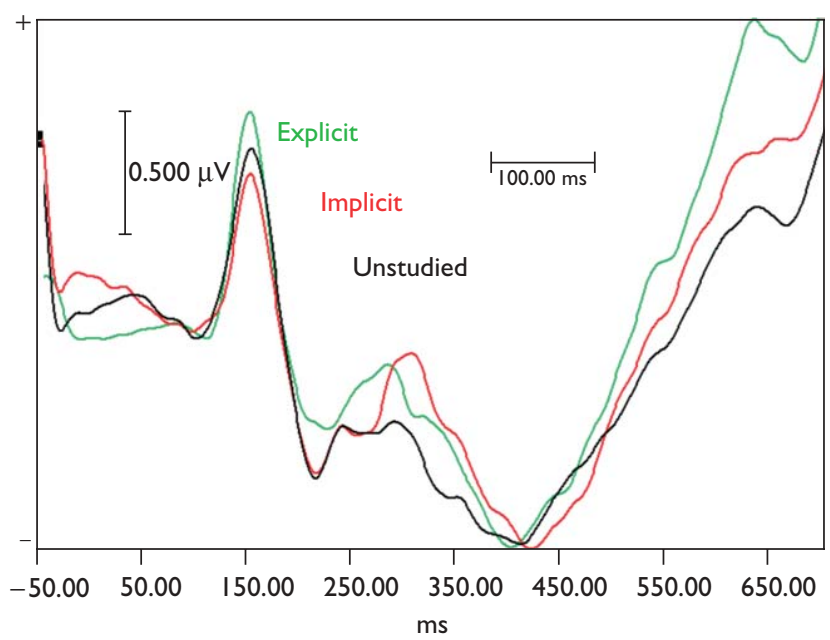

Fig. I Curves of global field power from event-related potential of three different tasks. 
200-400, 300-500 and 500-700 ms, in every channel. Then we performed multivariate analysis of variance for different tasks testing the interactions of locations $\times$ tasks with a Bonferroni post-hoc test. No significant intertask difference of areas under the curve was found between 100 and $200 \mathrm{~ms}$ and between 200 and $400 \mathrm{~ms}$ in all channels. For the explicit memory, scalp locations with statistical significance were mainly over either the parietal region between 300 and $500 \mathrm{~ms}$; for the implicit memory, significant locations were at the temporal-occipital areas between 300 and $500 \mathrm{~ms}$ and between 500 and $700 \mathrm{~ms}$ (Table 1).

In the topographic studies, we obtained t-Maps for the explicit memory from comparison of ERP between tasks with the well learned and unstudied figures; and t-Maps for the implicit memory between the unintentionally learned and unstudied figures. Finally, t-Maps for explicit versus

Table I Scalp locations with statistical significance in the memory eventrelated potential

\begin{tabular}{|c|c|c|}
\hline & $300-500 \mathrm{~ms}$ & $500-700 \mathrm{~ms}$ \\
\hline Explicit & $\begin{array}{c}\text { T3 }(0.013), \text { T5 }(0.014), \text { TP7 }(0.009), \\
\text { CP3 }(0.03 \mathrm{I}), \mathrm{P} 3(0.022), \mathrm{OI}(0.039) \\
\text { Pz }(0.04 \mathrm{I}), \mathrm{T} 4(0.047), \mathrm{T} 6(0.002), \\
\text { TP8 }(0.004), \mathrm{CP} 4(0.022), \mathrm{P} 4 \\
(0.005), \mathrm{O} 2(0.004)\end{array}$ & \\
\hline Implicit & P4 (0.036), O2 (0.004), OI (0.017) & $\begin{array}{c}\text { T6 (0.005), P4 (0.028), } \\
\text { O2 (0.0I), OI (0.006) }\end{array}$ \\
\hline
\end{tabular}

$P$ values from multivariate analysis of variance with Bonferroni post-hoc test for scalp locations $\times$ tasks on area under curve in every channel are given in parentheses. Explicit: well learned versus new material; implicit: unintentionally learned versus new material; electrode positions named after the 10-10 international nomenclature; as no significant difference was found between 100 and 200 ms and between 200 and 400 ms in all channels, it is not listed in the table. implicit memory were from comparison between the well learned and unintentionally learned figures. In the t-Maps of the explicit memory (Fig. 2a), the first significant activity appeared in the right occipitotemporal regions (around $275 \mathrm{~ms})$, soon extending to the left side $(325 \mathrm{~ms})(t>2.8$, $P<0.01)$. At about $400 \mathrm{~ms}$, a very prominent activation was noticed at the temporoparietooccipital regions of either hemisphere especially on the right side $(t>2.8, P<0.01)$. A small but significant $(t>2.1, P<0.05)$ left frontal activation occurred at $450 \mathrm{~ms}$ and later a right frontal activation occurred at around $500 \mathrm{~ms}(t>2.1, P<0.05)$. The t-Maps of the implicit memory (Fig. 2b) carried a similar spatialtemporal profile with the explicit memory except that there was no such significant left frontal activation $(450 \mathrm{~ms})$ or strong parietal spread $(400 \mathrm{~ms})$. All activities centered at $400 \mathrm{~ms}$ were much less than their explicit counterparts. The implicit memory has a very strong negativity at the temporoparietooccipital regions after $600 \mathrm{~ms}(t<-2.8$, $P<0.01)$. The t-Maps of explicit versus implicit memory (Fig. 2c) showed significant difference at the right temporal region around $475 \mathrm{~ms}(t>2.5, P<0.02)$.

\section{Discussion}

Whether the participants learned the figures intentionally or unintentionally, they can recognize the figures with almost no difficulty in either group. The visual memory tasks were purposely designed to obtain a ceiling effect of learning to ensure that the participants had learned all the necessary material before the ERP study. The two sets of figures, however, were quite differently learned in terms of awareness. The participants reported that they could mentally visualize the explicit learned material while the unintentionally learned material could be recognized only (a)

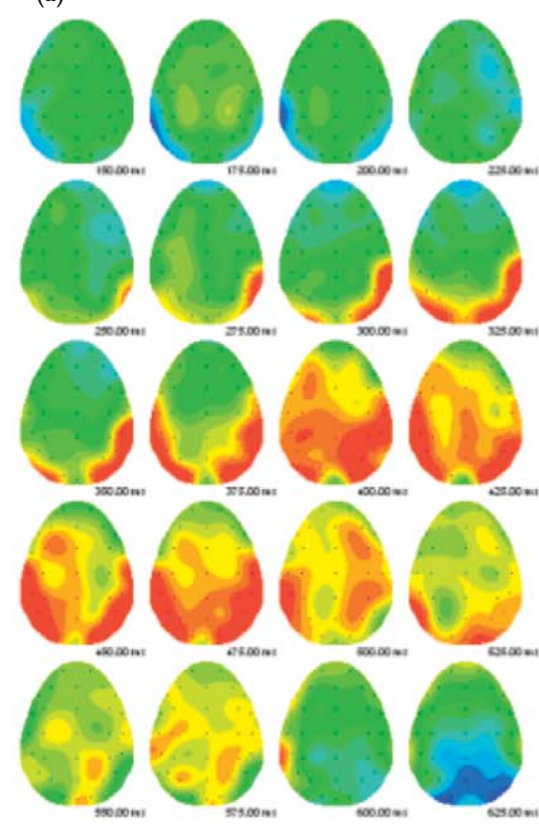

(b)

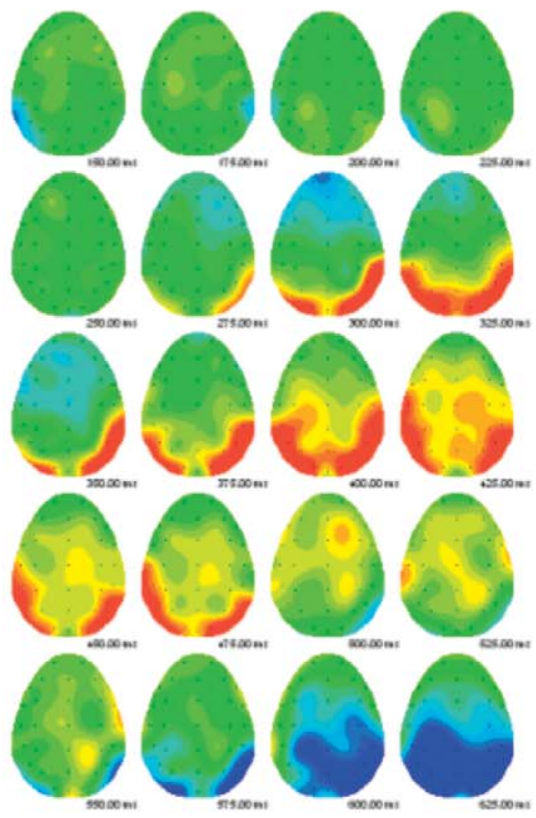

(c)

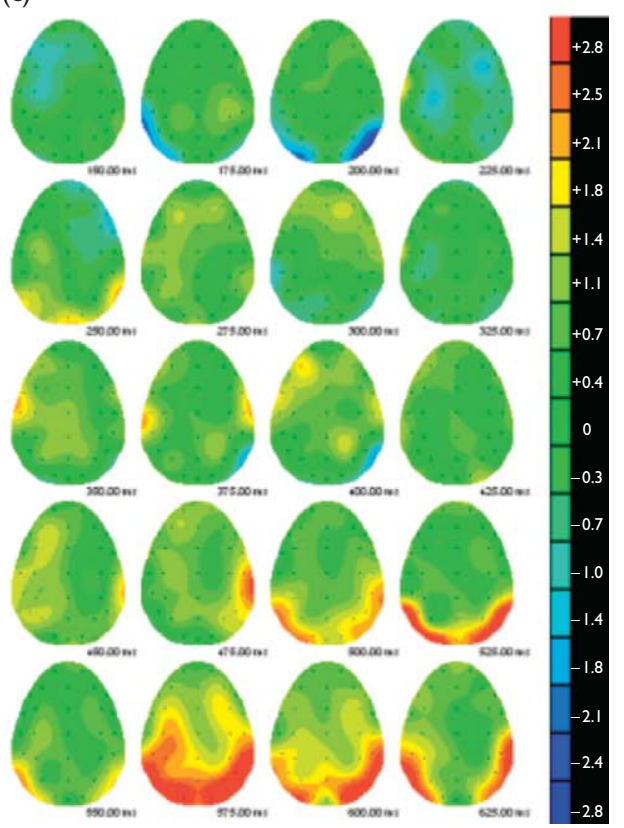

Fig. 2 (a) t-Maps for the explicit memory from comparison of event-related potential between tasks with the well learned and unstudied figures. (b) t-Maps for the implicit memory between the unintentionally learned and unstudied figures. (c) t-Maps for explicit versus implicit memory between the well learned and unintentionally learned figures. The color bar on the right side indicates the statistical significance levels: red for $P<0.01, t>2.8$, orange for $P<0.05, t>2$.I, deep blue for $P<0.01, t<-2.8$ and blue for $P<0.05, t<-2$.I. 
through a sense of familiarity. Thus, brain responses of pictorial learning with different levels of awareness could be differentiated through ERP compatible with a previous study [16].

Although an early effect of perceptual learning has been reported in the closure-related priming activity [17], in this study, the ERP around $150 \mathrm{~ms}$ was not significantly different; thus, it reflected merely an initial visual perceptual processing maximal at the occipital regions [18] but not related to memory function. The initial brain responses for either explicit or implicit memory effect of this study appeared at the right occipitotemporal region around 275$375 \mathrm{~ms}$. Activation of the visual associate cortex, that is, occipitotemporal regions, around $200-350 \mathrm{~ms}$ was interpreted as repetition-related processes for pictures or symbols, which might imply an encoding process essential for a further recognition memory process [19-21]. A prominent activation of the explicit memory at the parietal regions around $400-475 \mathrm{~ms}$ was observed in this study. This is the parietal old/new effect considered to be associated with recollection $[7,16]$. While processing the knowing but not memorized pictures, that is, the implicit memory, the parietal old/new effect around $400-475 \mathrm{~ms}$ was much less prominent than its explicit counterpart. The implicit memory relied on the perceptual priming-related mechanism occurring at the associate visual cortex, which was demonstrated in our study by persistent occipitotemporal activation (275- $475 \mathrm{~ms}$ ) compatible with the previous neuroimaging and electrophysiological studies [19,20,22]. As for the late positive component, the main effect appeared as a marked attenuation in the temporoparietooccipital areas after $600 \mathrm{~ms}$ (Fig. 2b). This is probably a consequence of the repetition suppression, that is, decreased strength of stimulus-related neuronal responses after repeated processing of identical stimuli in the extrastriate visual cortex [22].

The frontal old/new effect was also observed and it mainly occurred in the anterior frontal region while testing the explicit memory. Neuroimage evidence supported the crucial role of the anterior frontal regions in processing the studied words mainly with the remembering judgment and less with the knowing ones [23]. Frontal regions may contribute to the controlled processes that guided the retrieval and interacted with different posterior regions to complete the recollection process. To sum up with a previous ERP study for remembering and knowing, the maximal effects were over the parietotemporal and bilateral frontal electrode sites [24].

This study has successfully demonstrated dissociable forms of memory function with ERP, thus supporting the dual-process model of recognition memory. We presented the figures 12 times for the explicit learning tasks and six times for the implicit learning tasks before ERP study. Thus, the effect of memory strength could not be totally disregarded [12]. This is a limitation of our study.

\section{Conclusion}

Dissociable ERP patterns for recognition memory can be obtained through a carefully designed experiment. A controlled retrieval process of the frontal region interacting with the parietal recollection and the temporal encoding fulfill the explicit memory function. Occipitotemporal perceptual priming with repetition suppression in the extrastriate visual cortex characterizes the implicit memory function. ERP provides an insight into the dynamic process of the recognition memory function with an enhanced temporal resolution.

\section{References}

1. Shanks DR. Implicit learning. In: Lamberts K, Goldstone R, editors Handbook of cognition. London: Sage; 2004. pp. 202-220.

2. Gardiner JM, Richardson-Klavehn A. Remembering and knowing. I. In Tulving E, Craik FIM, editors. Oxford handbook of memory. New York: Oxford University Press; 2000. pp. 229-244.

3. Yonelinas AP. The nature of recollection and familiarity: a review of 30 years of research. J Mem Lang 2002; 46:441-517.

4. Jacoby LL. A process-dissociation frame-work: separating automatic from intentional uses of memory. J Mem Lang 1991; 30:513-541.

5. Rugg MD, Allan K. Event-related potential studies of memory. In: Tulving E, Craik FIM, eds. Oxford handbook of memory. New York: Oxford University Press; 2000. pp. 521-537.

6. Humphreys MS, Dennis S, Chalmers K, Finnigan S. Dual processes in recognition: does a focus on measurement operations provide a sufficient foundation? Psychonom Bull Rev 2000; 7:593-603.

7. Johnson R Jr. Event-related potential insights into the neurobiology of memory system. In: Boller F, Grafman J, editors. Handbook of neuropsychology. Vol. 10. Amsterdam: Elsevier; 1995. pp. 135-163.

8. Curran T. Brain potentials of recollection and familiarity. Mem Cogn 2000; 20:923-938.

9. Rugg MD, Mark RE, Walla P, Schloerscheidt AM, Birch CS, Allan K. Dissociation of the neural correlates of implicit and explicit memory. Nature 1998; 392:595-598.

10. Wagner AD, Gabrieli JDE. On the relationship between recognition familiarity and perceptual fluency: evidence for distinct mnemonic processes. Acta Psychol 1998; 98:211-230.

11. Wilding LW. In what way does the parietal ERP old/new effect index recollection? Int J Psychophysiol 2000; 35:81-87.

12. Finnigan S, Humphreys MS, Dennis S, Geffen G. ERP 'old/new' effects: memory strength and decisional factor(s). Neuropsychologia 2002; 40:2288-2304.

13. Curran T. The electrophysiology of incidental and intentional retrieval: ERP old/new effects in lexical decision and recognition memory. Neuropsychologia 1999; 37:771-785.

14. Lehmann D, Skrandies W. Reference-free identification of components of checkerboard-evoked multichannel potential fields. Electroencephalogr Clin Neurophysiol 1980; 48:609-621.

15. Skrandies W, Chiu MJ. Dimensions of affective semantic memory: behavioral and evoked potential correlates in Chinese subjects. Neurosci Letter 2003; 341:45-48.

16. Curran T, Cleary AM. Using ERP to dissociate recollection from familiarity in picture recognition. Cogn Brain Res 2003; 15:191-205.

17. Doniger GM, Foxe JJ, Schroeder CE, Murray MM, Higgins BA, Javitt DC. Visual perceptual learning in human object recognition areas: a repetition priming study using high-density electrical mapping. Neuroimage 2001; 13:305-313.

18. Treisman AM, Kanwisher NG. Perceiving visually presented objects: recognition, awareness, and modularity. Curr Opin Neurobiol 1998; 8:218-226.

19. Fiebach CJ, Gruber T, Supp GG. Neuronal mechanisms of repetition priming in occipitotemporal cortex: spatiotemporal evidence from functional magnetic resonance imaging and electroencephalography. J Neurosci 2005; 30:3414-3422.

20. Henson RN, Shallice T, Dolan R. Neuroimaging evidence for dissociable forms of repetition priming. Science 2000; 287:1269-1272.

21. Gruber T, Muller MM. Oscillatory brain activity dissociates between associative stimulus content in a repetition priming task in the human EEG. Cereb Cortex 2005; 15:109-116.

22. Henson RN, Rylands A, Ross E, Vuilleumeir P, Rugg MD. The effect of repetition lag on electrophysiological and haemodynamic correlates of visual object priming. Neuroimage 2004; 21:1647-1689.

23. Henson RN, Rugg MD, Shallice T, Josephs O, Dolan RJ. Recollection and familiarity in recognition memory: an event-related functional magnetic resonance imaging study. J Neurosci 1999; 19:3962-3972.

24. Duzel E, Yonelinas AP, Mangun GR, Heinze HJ, Tulving E. Event-related brain potential correlates of two states of conscious awareness in memory. Proc Natl Acad Sci USA 1997; 94:5973-5978. 